\title{
Increasing the One-Hop Progress of Nearest Neighbor Forwarding
}

\author{
Ralph Tanbourgi, Holger Jäkel, Friedrich K. Jondral
}

\begin{abstract}
Nearest neighbor forwarding (NNF) intends to maximize throughput in wireless networks. However, NNF suffers from low one-hop progress and may therefore significantly increase end-to-end delay. The spatial efficiency (SE), i.e., the expectation of the ratio of progress to interference area associated with one hop, is introduced in order to quantify this tradeoff. The problem of low progress is addressed by maximizing the one-hop SE, subject to the central angle $\gamma$, determining the forwarding area. By this, the optimal balance between minimizing the interference area and maximizing progress is found. Then, this analysis is extended by considering a Poisson point process, driven by some traffic intensity, on the interference area. Furthermore, the traffic aware $\gamma^{*}$-NNF strategy is proposed which adapts $\gamma$ to the traffic intensity in order to maximize SE. Simulation results show a significant reduction of the end-to-end delay if $\gamma^{*}$-NNF is used.
\end{abstract}

Index Terms-Wireless networks, greedy geographic routing, Poisson point process.

\section{INTRODUCTION}

W IRELESS networks have recently gained much attraction due to several reasons: research on hardware has achieved considerable advances in the development of small and inexpensive communications devices. A fundamental property of these networks is that communication does not rely on a wired backbone. Hence, nodes additionally have to function as routers by carrying the network traffic as well as to organize and to maintain network topology. Certain aspects of routing must therefore be reviewed and new design paradigms must be found in order to face emerging challenges such as scalability and mobility.

A potential approach is greedy geographic forwarding (GGF), which has gained much interest due to several reasons (see [1]-[3]). In GGF, forwarding decisions are based on local minimization of an Euclidean cost metric. This cost metric can have various forms [2], depending on the strategy of interest. In the GGF framework, nearest neighbor forwarding (NNF) represents a strategy that minimizes spatial contention and hence, maximizes throughput. In this scheme a packet is forwarded to the node which has the least Euclidean distance to the node currently holding the packet. By precondition, the selected node (or relay) must give a positive progress to the routing process, resulting in that only nodes located in a circle sector with central angle $\gamma=\pi$ are considered as potential relays. This circle sector is called the forwarding area and is indicated in Fig. 1(a).

The authors are with the Communications Engineering Lab (CEL) at the Karlsruhe Institute of Technology (KIT), Germany. Email: \{ralph.tanbourgi, holger.jaekel, friedrich.jondral\}@kit.edu

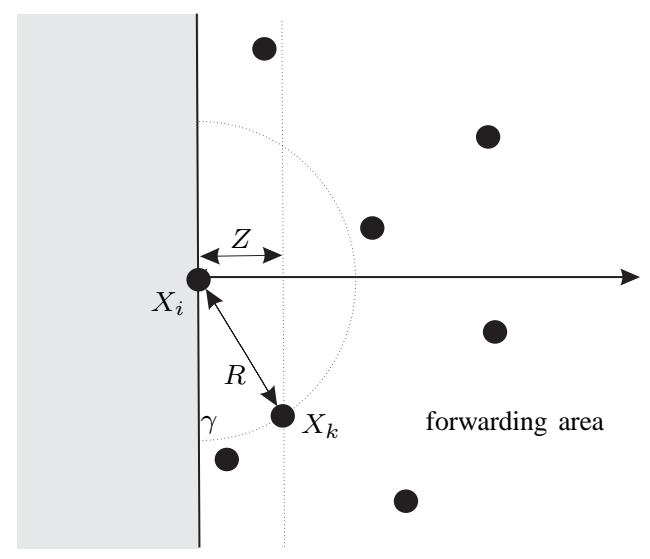

(a) Case I

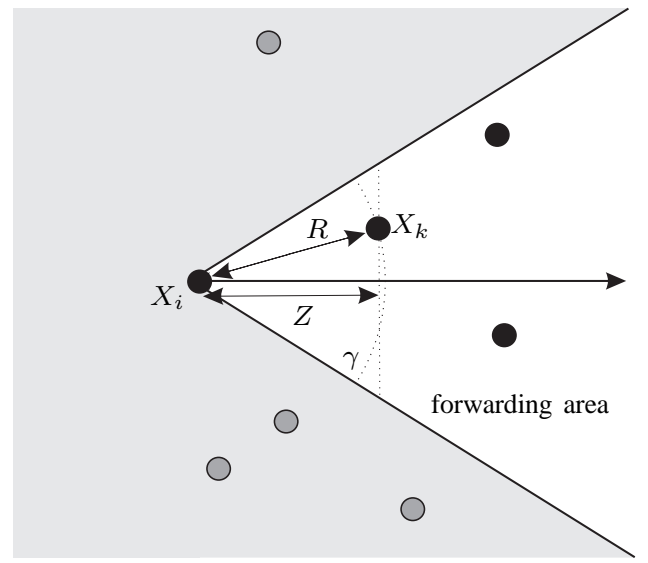

(b) Case II

Fig. 1. Case I: All nodes that give positive progress $Z$ are potential relays $(\gamma=\pi)$. Case II: Forwarding area is decreased $(\gamma<\pi)$ : Nearby nodes with low progress $Z$ are not considered as potential relays.

Although NNF maximizes throughput, it suffers from low progress which may cause high end-to-end delay. This problem can be tackled by narrowing the circle sector by means of $\gamma<\pi$ (Fig. 1(b)). Consequently, nearby nodes with low progress are not considered as potential relays anymore. This, of course, does not solve the problem, but decreases the probability of low one-hop progress and improves end-to-end delay. However, this modification results in more interference and hence, lower throughput due to the increased transmission power resulting from the increased one-hop distance.

We introduce the spatial efficiency (SE), which is defined as the expectation over the ratio of progress to interference area, in order to analyze this trade-off. Then, we determine 
the optimal central angle $\gamma^{*}$ such that the forwarding area is chosen to maximize SE. In the second step, we refine the definition of the SE by considering a Poisson point process (PPP) on the interference area. Then, we propose the traffic intensity aware $\gamma^{*}$-NNF strategy which adapts $\gamma$ to the traffic intensity in order to maximize SE. Finally, we present simulation results that reveal the significant reduction of endto-end delay if $\gamma^{*}$-NNF is used.

\section{Spatial EfFiciency OF NNF}

We assume that nodes are distributed in the plane according to a stationary PPP. All nodes are equipped with omnidirectional antennae. Furthermore, they do not have to obey a transmission power constraint ${ }^{1}$.

Denote by $R$ the transmission radius which is the Euclidean distance between source and relay. Furthermore, denote by $Z$ the one-hop progress which is obtained by projecting $R$ onto a line connecting source and destination. Then, we define the spatial efficiency (SE) as

$$
\eta:=\mathbb{E}\left\{\frac{Z}{R^{2}}\right\}
$$

where $\mathbb{E}\{\cdot\}$ is the expectation operator. The quantity $\eta$ measures the average one-hop progress normalized to the resulting interference area. The motivation for choosing this metric rather than, e.g., $\mathbb{E}\left\{\frac{Z}{R}\right\}$, is because it applies to the problem of spatial contention and throughput. A key assumption is that spatial contention is proportional to $R^{2}$ or equivalently, that throughput is inversely proportional to $R^{2}$, which we consider as the interference area associated with one hop. Since the nodes have omnidirectional antennae, the interference area is always a disc of radius $R$.

We now calculate $\eta$ for the NNF strategy for $\gamma$ being arbitrary but fixed. Since $Z$ and $R$ are dependent, we first decompose (1) by applying the law of total expectation, according to

$$
\eta=\mathbb{E}\left\{\frac{\mathbb{E}\{Z \mid R\}}{R^{2}}\right\}
$$

The probability density function (PDF) of $R$ is given by

$$
f_{R}(r)=\lambda \gamma r e^{-\lambda \frac{\gamma}{2} r^{2}}, \quad r \geq 0,
$$

where $\lambda$ is the node density of the PPP [4]. To calculate $\mathbb{E}\{Z \mid R\}$, we need the conditional PDF $f_{Z \mid R}(z \mid r)$. With the uniformity of the PPP, we can obtain $f_{Z \mid R}(z \mid r)$ by simple transformation of random variables, yielding

$$
f_{Z \mid R}(z \mid r)=\frac{2}{\gamma \sqrt{r^{2}-z^{2}}}, \quad r \cos (\gamma / 2) \leq z \leq r .
$$

With (4), the inner expectation in (2) can then be calculated as

$$
\mathbb{E}\{Z \mid R=r\}=\int_{r \cos (\gamma / 2)}^{r} \frac{2 z}{\gamma \sqrt{r^{2}-z^{2}}} \mathrm{~d} z=\frac{2 r}{\gamma} \sin (\gamma / 2) .
$$

\footnotetext{
${ }^{1} \mathrm{~A}$ constraint on transmission power would not give any additional insight to the trade-off involved in this work. Furthermore, limiting the transmission range would introduce connectivity issues to the model for fairness reasons.
}

Hence, (2) can be rewritten as

$$
\eta=2 \lambda \sin (\gamma / 2) \int_{0}^{\infty} e^{-\lambda \frac{\gamma}{2} r^{2}} \mathrm{~d} r=\sqrt{\frac{2 \lambda \pi}{\gamma}} \sin (\gamma / 2) .
$$

We maximize $\eta$ by letting $\frac{\partial \eta}{\partial \gamma} \stackrel{!}{=} 0$ s.t. $0<\gamma \leq \pi$, yielding

$$
\gamma^{*} \cos \left(\gamma^{*} / 2\right)-\sin \left(\gamma^{*} / 2\right) \stackrel{!}{=} 0, \quad \Rightarrow \gamma^{*} \approx 0.74 \pi .
$$

Note that the optimal $\gamma^{*}$ in (7) is independent of the node density $\lambda$.

\section{Poisson Point Process and Spatial Efficiency}

Now, we want to measure the SE in terms of the expected ratio of progress to the number of conflicts, according to

$$
\eta_{I}:=\mathbb{E}\left\{\frac{Z}{I+1}\right\},
$$

where $I$ denotes the number of conflicts. The term conflict refers to the problem of concurrent medium access in wireless network and thus characterizes either the degree of interference or the required number of orthogonal channels. We condition the PPP on having a node in the origin and count the number of conflicts this node experiences ${ }^{2}$. We further assume that interference is formalized by the protocol model [6].

Then, $I$ can be written as a PPP with intensity

$$
\Lambda_{I}=p \int_{\mathbb{R}^{2}} \mathbb{P}\{|x| \leq(1+\Delta) R\} \lambda \mathrm{d} x,
$$

where $p$ is the thinning factor, which can be seen as the network load and $\Delta$ denotes the guard zone around the receiver in the protocol model. We now condition $\Lambda_{I}$ on the fact that the transmission radius is $R=r_{0}$. Thus, (9) can be rewritten as

$$
\Lambda_{I}\left(r_{0}\right)=p \int_{\mathbb{R}^{2}} \mathbb{1}\left(|x| \leq r_{0}(1+\Delta)\right) \lambda \mathrm{d} x=\pi \lambda \beta r_{0}^{2},
$$

where $\beta:=p(1+\Delta)^{2}$ denotes the traffic intensity that drives the PPP. Again, we apply the law of total expectation and decompose (8), according to

$$
\eta_{I}=\mathbb{E}\left\{\mathbb{E}\{Z \mid R\} \mathbb{E}\left\{\frac{1}{I+1} \mid R\right\}\right\} .
$$

Note that conditioned on $R, Z$ and $I$ are independent. The second term is calculated as

$$
\mathbb{E}\left\{\frac{1}{I+1} \mid R=r\right\}=\frac{1-e^{-\pi \lambda \beta r^{2}}}{\pi \lambda \beta r^{2}} .
$$

With (5) and (12), (11) can be calculated as

$$
\eta_{I}=\frac{\sqrt{2} \sin (\gamma / 2)}{\beta \sqrt{\lambda \pi \gamma}}\left(1-\frac{1}{\sqrt{1+2 \beta \pi / \gamma}}\right)
$$

which is a function of $\beta$ and $\gamma$. We are now able to find the optimal $\gamma^{*}$ in terms of SE for a given traffic intensity $\beta$ which is the subject of the following section.

\footnotetext{
${ }^{2}$ Due to Slivnyak's theorem [5], this does not affect the distribution of the PPP.
} 


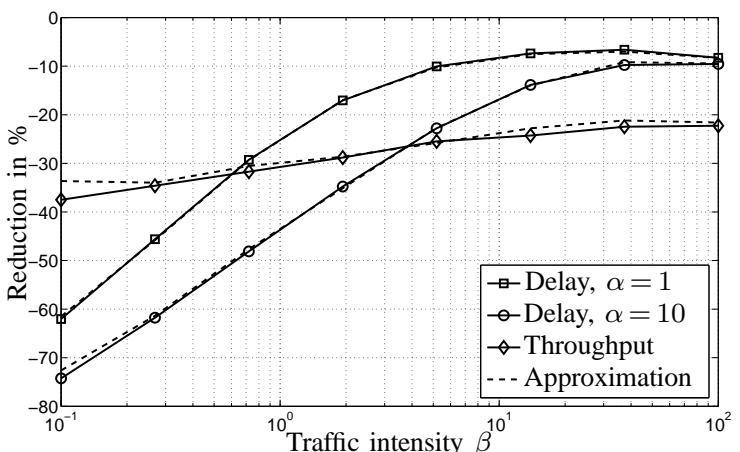

Fig. 2. Reduction of expected throughput and expected delay with respect to the case $\gamma=\pi$ vs. traffic intensity $\beta$ for different per-hop bias delays $\alpha$.

\section{TRAFFIC AWARE $\gamma^{*}$-NNF}

We assume that nodes have side information about the traffic intensity $\beta$, i.e., nodes are aware of the network load $p$ and of the decoding requirements respectively the guard zone $\Delta$. This can be achieved by, e.g., medium congestion measurements [7]. With this knowledge, nodes can compute the optimal central angle $\gamma^{*}(\beta)=\arg \max _{\gamma}\left\{\eta_{I}(\gamma, \beta)\right\}$ from (13). Unfortunately, $\gamma^{*}(\beta)$ can not be obtained in closedform. Since $\lim _{\beta \rightarrow \infty} \gamma^{*}(\beta)=0.74 \pi$ and by considering only a network load $p>0.15$, i.e., interference-limited networks, we can approximate $\gamma^{*}(\beta)$ by curve fitting yielding

$$
\gamma^{*}(\beta) \simeq \begin{cases}0.14 \pi, & 0 \leq \beta<0.15 \\ -1.16 \beta^{-\frac{1}{3}}+2.64, & 0.15 \leq \beta \leq 50 \\ 0.74 \pi, & \beta \geq 50 .\end{cases}
$$

We conducted Monte Carlo simulations to analyze the resulting reduction in delay. In the scenario, nodes were i.i.d. distributed with density $\lambda=10^{-3}$ in an area of $1000 \times 1000$ $\mathrm{m}^{2}$ and a source-destination pair was placed with distance $10^{3}$ meters. We then measured the total delivery time of one packet from source to destination. Thereby, in each hop the number of concurrent medium access $I$ experienced by the forwarding node was measured using the protocol model. The per-hop delay was modeled as the sum of the waiting time in terms of multiple time slots due to TDMA queueing, of signal propagation duration and of a fictive bias per-hop delay $\alpha$ due to signal processing tasks, i.e., per-hop delay is $I+1+\alpha$ time slots. The simulations were conducted $10^{3}$ times for every $\beta$.

Fig. 2 shows the reduction of the expected end-to-end delay and the expected throughput vs. $\beta$ for $\gamma=\gamma^{*}$ (14). The solid lines and the dashed lines are with respect to the numerical maximization of (13) and the approximation from (14). It can be seen that the expected end-to-end delay can be reduced significantly in the low traffic regime (30-70\%). In the high traffic regime, the reduction of the expected end-to-end delay is approximately $10 \%$. Since the cost of reducing delay is an increase in interference area, we observe a reduction of the expected throughput (20-35\%) over the whole range of $\beta$. Furthermore, the approximation error when using (14) is marginal as observed in Fig. 2.

Fig. 3 shows the delay ratio and the throughput ratio for different non-optimal $\gamma$ with respect to using $\gamma^{*}$ vs. $\beta$. In this

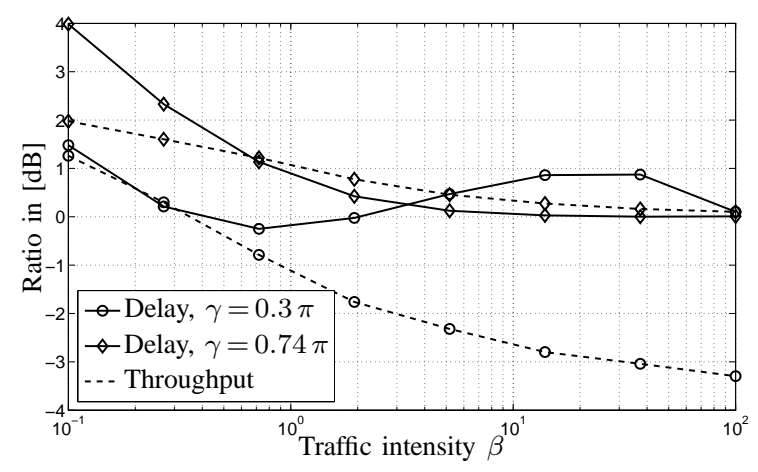

Fig. 3. Delay ratio and throughput ratio for non-optimal $\gamma$ with respect to $\gamma^{*}$ vs. traffic intensity $\beta$ for a per-hop bias delay $\alpha=5$.

scenario, $\gamma^{*}$ was computed numerically. It can be observed that using a static $\gamma$ results in a unstable delay-throughput trade-off: depending on the value of $\gamma$, either delay increases in the low traffic regime or throughput decreases in the high traffic regime compared to the optimal $\gamma^{*}$ case.

\section{CONCLUSION}

We addressed the problem of low progress of NNF, which may result in high end-to-end delay in wireless networks. We formulated the spatial efficiency as the expectation of the ratio of progress to interference area. By maximizing the spatial efficiency we obtained the optimal central angle $\gamma^{*}=0.74 \pi$, which finds the optimal trade-off between high progress and low spatial contention. We extended this analysis by considering a Poisson point process, driven by some traffic intensity, on the interference area associated with one hop. The protocol model was chosen as an example for interference characterization but can be replaced by other models. We proposed a new traffic aware forwarding scheme called $\gamma^{*}$ NNF which adapts its forwarding strategy to maximize the spatial efficiency for a given traffic intensity. In terms of end-to-end delay and throughput, our results show significant improvements compared to conventional NNF. Simulation results show that in this case, end-to-end delay can be reduced significantly (10-75\%), while throughput decreases only marginally (20-35\%).

\section{REFERENCES}

[1] M. Mauve, J. Widmer, and H. Hartenstein, "A survey on position-based routing in mobile ad-hoc networks," IEEE Network, vol. 15, pp. 30-39, 2001.

[2] I. Stojmenovic, "Position-based routing in ad hoc networks," IEEE Communications Magazine, vol. 40, no. 7, pp. 128-134, July 2002.

[3] C. Lemmon, S. M. Lui, and I. Lee, "Geographic forwarding and routing for ad-hoc wireless network: A survey," in INC, IMS and IDC, 2009. NCM '09. Fifth International Joint Conference on, 2009, pp. $188-195$.

[4] M. Haenggi, "On distances in uniformly random networks," Information Theory, IEEE Transactions on, vol. 51, no. 10, pp. 3584 -3586, 2005.

[5] M. Haenggi, J. Andrews, F. Baccelli, O. Dousse, and M. Franceschetti, "Stochastic geometry and random graphs for the analysis and design of wireless networks," Selected Areas in Communications, IEEE Journal on, vol. 27, no. 7, pp. 1029-1046, September 2009.

[6] F. Xue and P. R. Kumar, Scaling Laws for Ad Hoc Wireless Networks: An Information Theoretic Approach, ser. Foundations and Trends in Networking. Now Publishers, vol. 1, no. 2.

[7] Y. Chun Hu and D. B. Johnson, "Exploiting congestion information in network and higher," in In: Proc. of the 24th Intl Conf. on Distributed Computing Systems, 2004, pp. 301-310. 